\title{
Optimisation of Renewable Energy Systems
}

\author{
K. Mitchell M. Nagrial J. Rizk \\ *School of Engineering and Industrial Design \\ University of Western Sydney \\ PO Box 10, Kingswood, NSW 2747 \\ AUSTRALIA \\ E-mail: k.mitchell@uws.edu.au
}

Keywords: Renewable Energy, Optimisation, Simulation.

\begin{abstract}
Renewable energy systems are still much more expensive than traditional grid connected power, except in remote areas. Thus it is important that designs be optimised to achieve the least delivered energy cost over a lifetime of 20 years or so. This paper shows that to achieve grid level of reliability with wind solar and storage units alone is impossibly expensive so practical designs have always incorporated standby generation of some sort or other. This paper describes a simulation technique that enables a cost minimal, balanced system design.
\end{abstract}

\section{Introduction}

Many previous papers e.g. [1,2] have discussed optimisation of renewable energy systems involving wind, solar, storage and standby engine components. The optimisation process was achieved by developing probability density functions (pdf's) for the load, solar and wind outputs. Whilst mathematically correct, the drawback in these earlier works was the requirement to have precise hourly records of wind and solar outputs at a site near where the system was to be installed. Such records rarely exist in Australia, although more general records, based on daily data are available. In this work, a different approach involving simulation has been adopted to solve these difficulties.

A renewable energy system (RES) model has been set up that uses actual wind, solar and load data available over a 3-year period in western Sydney. The advantage of the modelling approach is that the impact of varying the main design parameters such as wind, solar and storage capacity on system availability can be readily determined and for a given performance outcome, the mix of wind, solar and storage capacities that gives the optimum cost can also be determined experimentally. A further advantage is that the inherent relationships between system loading and wind and solar outputs are not compromised.

The main disadvantage of the simulation approach is that ideally, many simulation runs should be performed to ensure that the top $1 \%$ of the "tails" of the distributions would be adequately covered, as it is these "tails" that determine system reliability.

\section{Description Of System Model}

The RES model used contained wind, solar, energy storage, AC-DC conversion, and local (AC) load components. The wind energy conversion system and the solar panels provided the renewable energy inputs and batteries the energy storage. The modelling covered stand-alone conditions, with and without back up generation. The model, named "Renewable System Simulator", is set up on an Excel spreadsheet with embedded Visual Basic macros. The model takes load, wind and solar data, and hourly load patterns. The model is set up to do three separate 28-day simulation runs in each system study (refer to the Addendum), this being geared to available data. However, there is nothing inherent in the software that would prevent multiple runs of arbitrary length being analysed.

In this study, three simulation runs were chosen as this allows the main seasonal variations to be modelled in the system in a single study in Sydney, as the region's annual wind/solar patterns seem to best fit a three-season, rather than the traditional four-season year. Readily available meteorological data is used. Wind or especially solar insolation data is rarely available in hourly levels, so pseudohourly data has had to be constructed for the analysis.

\subsection{Wind Data}

Weather data was obtained from the Australian Bureau of Meteorology in a standard format, which included total "bright sunshine hours", and eight sets of 3-hourly average wind speeds and directions. In the model, the 3-hour wind data was approximated to one-hour data by assuming it remained constant for the full 3 hours. Average 3-hourly wind speeds for the western Sydney area for the study period are shown in figure 1 .

From the wind speed data, wind turbine output is calculated from the turbine speed-power characteristic, i.e. 
$V w<$ Vcutin: $\rightarrow$ Pwr $=0$

Vcutin $\leq V w<$ Vrated $: \rightarrow P w r=$

$P_{\text {_ rated }}($ Vw - Vcutin $) /($ Vrated - Vcutin $\left.)\right]$

Vrated $\leq V w<$ Vshut - down: $\rightarrow P w r=P$ _rated

$V w \geq V$ shut - down $: \rightarrow P w r=0$

where -

Power = the power output of the wind turbine,

$\mathrm{V}_{\mathrm{w}}=$ the wind speed, assumed constant for the hour,

$\mathrm{P} \_$rated $=$the nominal rated power of the turbine,

$\mathrm{V}_{\text {cutin }}=$ the cut-in speed of the turbine,

$\mathrm{V}_{\text {rated }}=$ the rated speed of the turbine,

$\mathrm{V}_{\text {shut-down }}=$ the shut-down speed of the turbine.

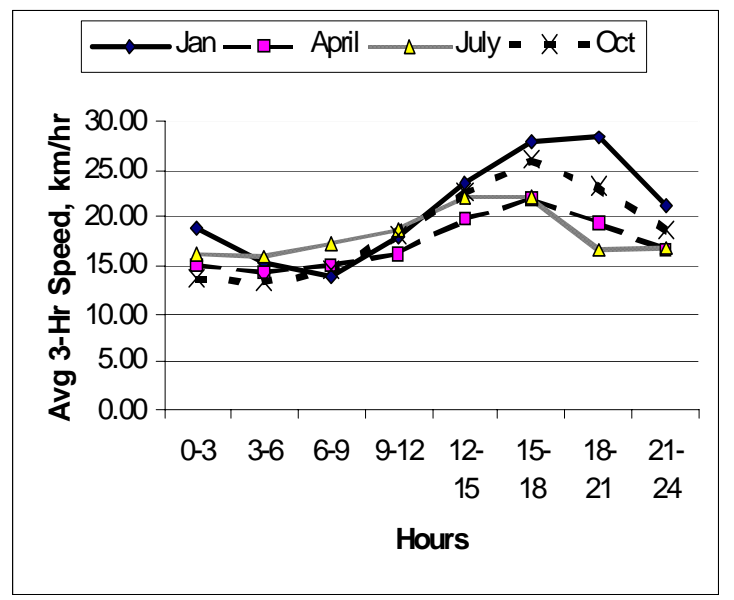

Figure 1 - Average 3-Hourly Wind Speeds

\subsection{Solar Data}

Detailed hourly solar data for the period of interest was not available. The most common form of data is the so-called "bright sunshine hours", the daily total of hours each day during which the sun is strong enough to burn a trace on a standard Campbell-Stokes recorder.

While this sort of data can give a good idea of total daily energy, it is of less direct help to model hourly variations. However, average beam and diffuse solar radiation levels by month for a horizontal plane was available for Sydney in one location. This data was used to develop a correlation between Daily BSS Hours and hourly distributions and an empirical relationship was developed, to create average hourly distributions from the BSS data. Distributions of total daily "bright sunshine hours" (BSS) by season, for the western Sydney area over the study period are shown in figure 2 .

\subsection{Equations}

The empirical formula used to generate the pseudo-hourly solar distributions from the BSS Hours data is listed below. It is a reasonable assumption to take the "beam" or direct radiation as a proxy for "bright” sunshine.

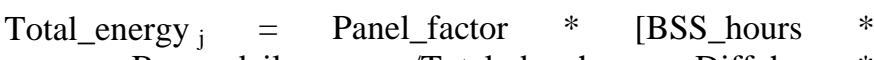
Beam_daily_energy/Total_day_hours + Diff_hours * Diff_daily_energy / Total_day_hours]

Energy $_{\mathrm{i}, \mathrm{j}}=$ Average $_{\mathrm{i}} *$ (Total_energy $_{\mathrm{j}} /$ Average total)

Where,

Beam_daily_energy = Month_peak_equivalent hours * 1.1* Noon_panel_radiation

Diff_daily_energy $=$ Month_peak_equivalent hours * $0.2 *$ Noon_radiation

Panel_factor $=$ Panel peak output at $1,000 \mathrm{~W} / \mathrm{m} 2$ (in $\mathrm{kW}$ )

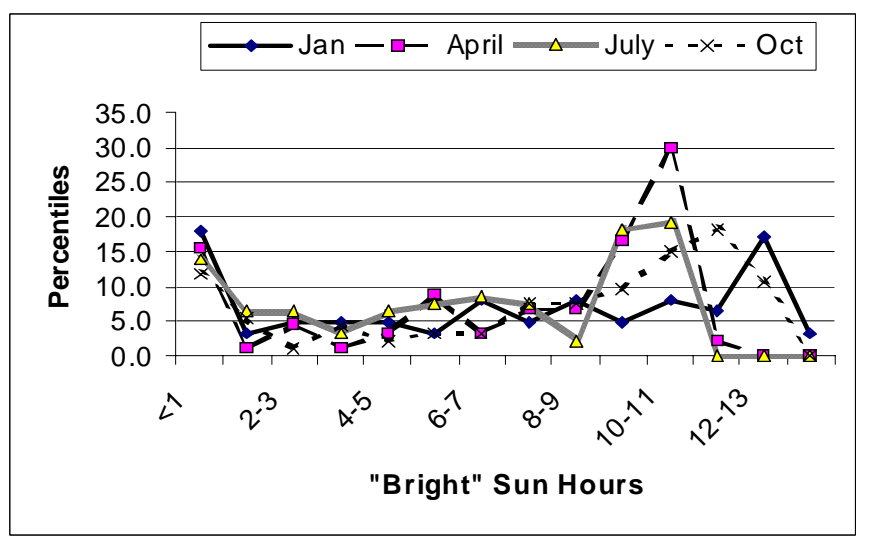

Figure 2 - Distribution of Daily Bright Sunshine Hours

\subsection{Load Data}

The load of course varies considerably from user to user. However, given that the objective is to model typical user load, data averaged from "zone" substations in the area was used. The latter supply typically 10,000-15,000 residential customers (or equivalent) each. A pseudo single customer daily load cycle can be obtained from this whole substation, by a pro-rata reduction of total substation hourly load to the average daily per customer energy consumption in NSW. Thus, the per customer average load cycle multiplied by total customers was equated to the total substation load. Two standard daily load cycles were adopted: one for summer and the second, for winter/spring. These were again based on average data for the zone substations. The substation chosen for the simulations was in the Glenmore Park area, near Penrith in western Sydney, where an automatic weather station with publicly available wind and bright sunshine hours data is nearby. To derive hourly loading on a day-by-day basis, total zone substation hourly energy was pro-rated based on the "standard" load cycles and average daily energy, according to eqn. 3 :

Load ${ }_{i, j}=$ Average $_{i} *\left(\right.$ Total $_{j} /$ Average total $)$ ... (3),

where -

$\operatorname{Load}_{\mathrm{i}, \mathrm{j}} \quad=$ the energy (hourly load) for hour ' $\mathrm{i}$ ' on day ' $\mathrm{j}$ ' 
Average $_{\mathrm{i}}=$ the average load cycle load at hour 'i' for that season

Total $_{\mathrm{j}} \quad=$ the actual total energy for day

'j'

Average total $=$ the average daily total energy

for that season.

\section{Simulation Runs}

\subsection{No Standby Plant}

The first study assumed full renewable energy autonomy, with no standby at all. Main system parameters (wind, solar and battery storage capacity) were varied to determine impact on system availability. The model did not calculate availability directly, but instead calculates the minimum BSOC in any 28-day simulation run, this being an effective proxy. A minimum permissible BSOC of $30 \%$ was set.

The "worst" season will depend on the net effect of wind and solar inputs and load, and of course, will be very location specific. In the Sydney area, load is highest in winter and summer and lowest in spring and autumn; solar outputs are least in winter and highest in spring/early summer, as are wind outputs. Thus spring is the season with greatest excesses of renewable inputs over load, whilst winter has the least inputs compared to load and is thus the "worst" season.

In winter, the minimum BSOC was reached after several days without standby plant. This was an event wherein a sequence of several days of low sun and wind output occurs, followed by days of higher outputs.

Wind, solar and load data was obtained for the period 19972000 in the Penrith area of Western Sydney. The results of the modelling under varying wind, solar and battery storage capacities are summaries in figure 3 . The curves are based on the simulation for spring data, under full autonomy (i.e. no standby).

Figure 3 - Minimum BSOC for Various Combinations of

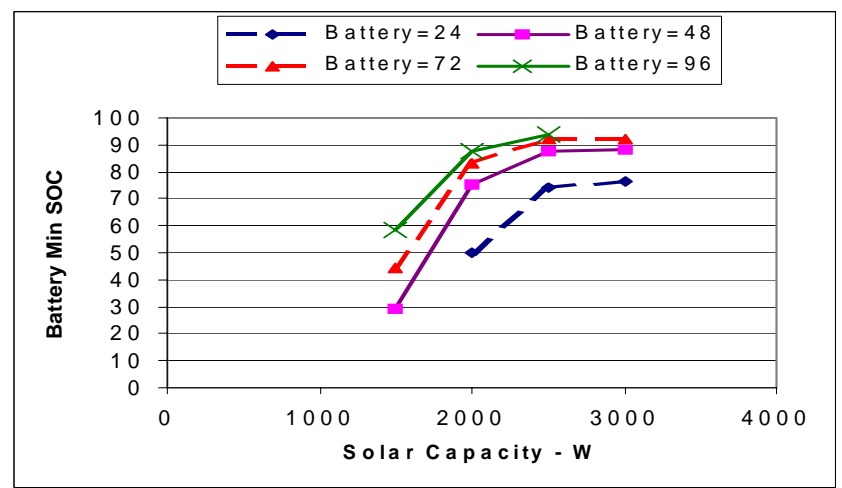

Wind, Solar and Storage Capacities

The rapidly diminishing benefit of increased wind/solar module ratings and storage capacity is evident, with a "natural” limit of about 90\% minimum BSOC displayed by the "kneeing" of the curves in figure 4. This relates to solar and wind capacities of $2 \mathrm{~kW}$ (or approximately 2 x peak load) and a storage capacity of $50 \mathrm{kWh}$, or approximately 2.5 average daily energy. This point however gives a system "reliability" (strictly speaking, availability) well below grid reliability standards. Thus any practical RES must have standby plant to be at all cost optimal. As a comment on seasonality, with the same model, for simulation runs on summer and spring data resulted in the minimum BSOC is about $85 \%$.

It is also interesting to note that (at least in the Sydney region), the energy input from a solar panel with the same nominal peak rating as a wind turbine will produce about $50 \%$ more energy. The results for optimum sizing are shown in table 1, for the simulation run for spring in the Full Autonomy study.

Table 1 - Optimum Sizing Spring (no Backup Plant)

\begin{tabular}{|l|l|l|l|}
\hline Wind (W) & 2000 & 2000 & 2000 \\
\hline Solar (W) & 1500 & 2000 & 2500 \\
\hline Battery (kWh) & 120 & 48 & 48 \\
\hline Min BSOC \% & 66.56 & 66.72 & 76.03 \\
\hline Cost (\$) & 38,630 & 35,612 & 40,235 \\
\hline
\end{tabular}

In the case of the system modelled, with average daily energy usage of $17 \mathrm{kWh}$ and average daily peak of $1.5 \mathrm{~kW}$, the optimum costs for a satisfactory (minimum BSOC > 60\%) performance for spring was with peak ratings of $1500 \mathrm{~W}$ and $2000 \mathrm{~W}$ in the solar and wind modules, respectively and an energy storage of $72 \mathrm{kWh}$ (about 4 days). To be completely autonomous through winter required peak ratings of $3000 \mathrm{~W}$ in both the solar and wind modules, respectively and energy storage of $192 \mathrm{kWh}$. System cost was \$76,000 at an average energy cost of $\$ 0.70$ per $\mathrm{kWh}$ over 20 years. The increase is quite remarkable and highlights the cost issue of attempting to design fully autonomous systems.

\subsection{With Standby Plant}

The second study was a repeat of the first but this time it was assumed standby plant was available. Standby avoids system unavailability (aside from component failures), but can result in wastage of the renewable energy resource, if the standby plant is switched in to recharge batteries only to be immediately followed by excess wind or solar inputs (see “spilt energy", sect 3.3).

With stand-by plant, energy input occurs when BSOC falls below a permissible minimum. The immediate design impact is to allow smaller wind/solar capacities and energy storage. The stand-by engine in these studies was rated at 2000W. The same simulation as before was used, thus the same weather load data applied; the only difference between the two simulations being the presence of the standby plant. 
With the same system, the minimum BSOC for simulation runs in summer and spring data was about $85 \%$. The results are identical whether with standby or no standby, the reason being that there is so much available renewable energy in spring and summer that standby plant is never required in those seasons.

Including the cost of the standby plant and fuel, the optimum cost is obtained when allowing some standby operation at most times during winter. The optimum sizing, using a fuel cost of $\$ 0.30$ per kWh was with capacities of $500 \mathrm{~W}, 1000 \mathrm{~W}$ and $24 \mathrm{kWh}$ for the wind, solar and energy storage modules. This is considerably smaller than with no standby plant allowing for an overall cost reduction. The effect is shown in table 2, where the lowest system cost was $\$ 40,000$ at an average energy cost of $\$ 0.39$ per kWh over 20 years.

Table 2 - Optimum Sizing with Back-up Plant

\begin{tabular}{|l|l|l|l|}
\hline Wind (W) & 1000 & 500 & 500 \\
\hline Solar (W) & 1000 & 1000 & 500 \\
\hline Battery (kWh) & 24 & 24 & 24 \\
\hline Min BSOC \% & 30 & 30 & 30 \\
\hline Cost (\$) & 40,000 & 39,400 & 41,700 \\
\hline
\end{tabular}

This reinforces the fact that for a practical system, some standby power must be included to meet needs of a real-sized house in all seasons in a mid latitude climate.

\subsection{Standby Plant with a Predictive Controller}

The model allows the use of both standard set point and "predictive" controllers as regards switching of stand-by plant. This is related to the phenomenon of "spilt" energy. Stand-by engines are best run continuously at full throttle once switched on, for engine life and fuel efficiency maximisation. With a set-point controller under this regime, the battery would be allowed to charge up to $100 \%$ state of charge (or some other predetermined value). If a day with high solar/wind inputs should follow a stand-by charging run, the renewable energy input is wasted, as the batteries are already at $100 \%$ BSOC, and is "spilt". This of course represents wasted money and excess expensive imported standby energy. The mechanism is shown in figure 4.

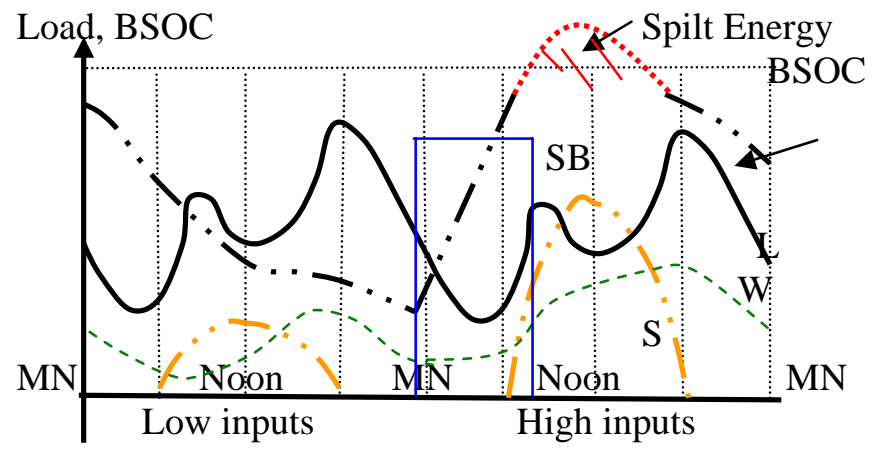

Figure 4 Daily Energy Flows - Low Net Inputs
For high load days, or low solar/wind input days, the battery will not receive enough energy and will progressively discharge over days. In these cases the stand-by plant will come in on-line "(SB)", which shows the case where a day of low inputs is followed by a day of high inputs. The plant will normally be run at full power until the battery reaches full BSOC and the stand-by plant will cut out. If a day of relatively high renewable energy input should follow, this "free" energy will be wasted, as the battery storage is already full. The cross-hatching shows the resulting "spilt" energy.

It was found that reduction of the cutout point from $100 \%$ to about $85 \%$ BSOC reduced spilt and standby energy, as more energy buffer is available. Reducing the cut-out point below this figure does not assist any further however, and in the case of a much lower cut-out point (e.g. < 70\%), stand-by energy begins to increase, as more switching of the standby plant is required. One method to further reduce the standby and spilt energy is to replace a simple set-point controller with an "intelligent" one that attempts to predict incoming wind and solar energy.

A number of papers have been written on this subject, e.g. Lawrence et al [3] wherein solar input was predicted, based on clear sunny conditions, and various short-term windforecasting algorithms have been developed. The latter require considerable computing power whereas what is desired for small standalone systems is a simple, cheap controller. The "predictive controller" modelled in this study uses seasonal average daily load, solar and wind energy flows to pre-determine what amount of renewable energy could be expected at any hour of the day for average conditions. This energy is subtracted from $100 \%$ to determine a recommended cutout BSOC point below 100\%.

The method of determining the expected renewable inputs at any time of day is shown in figure 5. The maximum amount of input energy to allow for is the maximum positive net energy that will occur at any hour ahead during the next 24 hours.

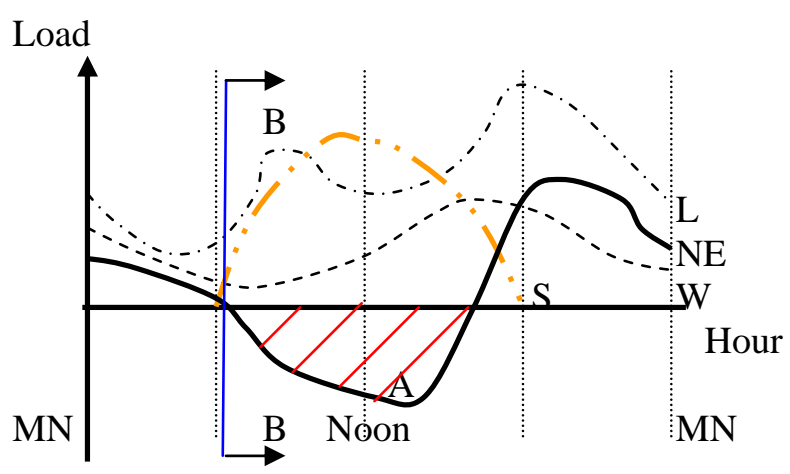

Figure 5 Determination of Expected Inputs

This can be net input, e.g. shaded area ' $A$ ' on the net energy curve NE when looking head from hour ' $\mathrm{i}$ ' (B-B), or a net load, when at other times. When at hour ' $\mathrm{i}$ ', the maximum expected over the next few hours is positive, so the battery 
charging cut-out BSOC will be discounted from 100\% by this amount of energy ('A'), to avoid spilling.

The algorithm is, at hour i:

$(\text { Expected input })_{\mathrm{i}}=\operatorname{Max}\left\{\Sigma^{24}{ }_{1 \neq \mathrm{i}}(\text { Net load })_{\mathrm{i}}\right\}$

$(\text { Cut-out SOC \%) })_{\mathrm{i}}=100-$ Factor $*(\text { Expected input })_{\mathrm{i}}$ ...(3.7)

The variable "Factor" allows for high input days where energy will exceed averages. Such variation will be driven by the net energy probability distribution, which is the convolution of the load, wind and solar energy distributions. The optimum value of "Factor" has been found to be $25 \%$ by experiment rather than by theoretical means. The predictive routine is compared to a set-point controller for the same load, wind and solar data. Savings of up to $15 \%$ can be observed, depending on days of storage, as shown in table 3. Savings decrease as storage capacity increases, owing to the larger buffer and disappears after about 3 days’ storage.

Table 3 - System Imported Energy, kW (28 days)

\begin{tabular}{|l|l|l|l|l|}
\hline Storage & 1 day & 1.5 days & 2 days & 3 days \\
\hline $\begin{array}{l}\text { Set-point } \\
100 \%\end{array}$ & 249 & 223 & 199 & 187 \\
\hline Predictive & 206 & 197 & 188 & 186 \\
\hline \% Savings & 17 & 12 & 5.5 & 0.5 \\
\hline
\end{tabular}

\section{Conclusions}

The findings of modelling studies done to date can be summarized as follows:-

- $\quad$ System minimum BSOC and hence availability generally increases with increasing wind/solar capacity and energy storage

- A certain minimum wind/solar output is needed to satisfy long-term average load, regardless of storage

- A certain minimum storage is needed to satisfy shortterm output deficiencies, regardless of capacities, because near-zero output days (windless, cloudy) do occur, usually as a sequence,

- System behaviour is related to wind/solar/load probability distributions and is very location specific,

- Increasing energy storage or wind/solar device outputs above the "natural" levels does little to improve system availability, suggesting that there are natural limits to system availability,

- The long "tails” of the PDF's mean that very high levels of availability is uneconomic and therefore stand-by plant will always be imperative in a sensible design,

- "Predictive" controllers in RES's can reduce the amount of "split" energy and hence standby power drawn by about $15 \%$

\section{References}

[1] Borowy S B, Salameh Z M, "Methodology for Optimally Sizing the Combination of a Battery Bank and PV Array in a Hybrid Wind/PV System”, IEEE Transactions on Energy Conversion, Vol 11, No 2, (June 1996)

[2] Abouzahr I, Ramakumar R, "Loss of Power Supply Probability of Stand-alone Wind Electric Conversion Systems”, IEEE Transactions on Energy Conversion, Vol 5, No 3, (Sep 1990)

[3] Wichert B, Lawrance W, Friese T, "First Experiences with a Novel Predictive Control Strategy for PV-Diesel Hybrid Energy Systems”, Solar'99 (1999) 